\title{
Descending Dungeons and Iterated Base-Changing
}

\author{
David Applegate, Marc LeBrun and N. J. A. Sloane
}

To OUR FRIEND AND FORMER COlleague Peter FishbuRn, ON THE OCCASION OF HIS 70TH BIRTHDAY.

\begin{abstract}
For real numbers $a, b>1$, let $a_{b}$ (also written as $a_{-} b$ ) denote the result of interpreting $a$ in base $b$ instead of base 10. We define "dungeons" (as opposed to "towers") to be numbers of the form $a_{-} b_{-} c_{-} d_{-} \ldots_{-} e$, parenthesized either from the bottom upwards (preferred) or from the top downwards. Among other things, we show that the sequences of dungeons with $n^{\text {th }}$ terms $10 \_111_{-} 2_{\ldots} \ldots(n-1) \_n$ or $n_{-}(n-1) \ldots \ldots+12 \_11 \_10$ grow roughly like $10^{10^{n \log \log n}}$, where the logarithms are to the base 10. We also investigate the behavior as $n$ increases of the sequence $a_{-} a_{-} a_{-} \ldots{ }_{-} a$, with $n a$ 's, parenthesized from the bottom upwards. This converges either to a single number (e.g. to the golden ratio if $a=1.1$ ), to a two-term limit cycle (e.g. if $a=1.05$ ) or else diverges (e.g. if $a=\frac{100}{99}$ ).
\end{abstract}

Keywords: towers, dungeons, sequences, recurrences, discrete dynamical systems

2000 Mathematics Subject Classification: 11B37, 11B83, 26A18, 37B99

David Applegate

AT\&T Shannon Labs, 180 Park Ave., Florham Park, NJ 07932-0971,

e-mail: davideresearch.att.com

Marc LeBrun

Fixpoint Inc., 448 Ignacio Blvd. \#239, Novato, CA 94949, e-mail:mlbewell.com

N. J. A. Sloane

AT\&T Shannon Labs, 180 Park Ave., Florham Park, NJ 07932-0971,

e-mail: njas@research.att.com 


\section{Introduction}

The starting point for this paper was the question: what is the asymptotic behavior of the sequences

$$
\begin{aligned}
& 10,1_{11},{ }^{10_{11_{12}}},{ }^{10_{11_{12}}}, \ldots, \\
& 10,11_{10}, 12_{11_{10}},{ }^{13_{12}}{ }_{11_{10}}, \ldots,
\end{aligned}
$$

where, for real numbers $a, b>1, a_{b}$ (or, more conveniently although less graphically, $a_{-} b$ ) denotes the result of interpreting $a$ in base $b$ instead of base 10 ? That is, if $a$ is a real number $>1$, with decimal expansion

$$
a=\sum_{i=-\infty}^{k} c_{i} 10^{i}, \quad \text { for some } k \geq 0, \text { all } c_{i} \in\{0,1, \ldots, 9\}, \text { and } c_{k} \neq 0,
$$

and $b$ is a real number $>1$, then

$$
a_{b}:=a_{-} b:=\sum_{i=-\infty}^{k} c_{i} b^{i} .
$$

We use text-sized subscripts in expressions like $a_{b}$ to help distinguish them from symbols with ordinary subscripts. The sum in (3) converges, since

$$
1<a_{b}<9 b^{k+1} /(b-1),
$$

and $a_{b}$ is well-defined if we agree to avoid decimal expansions ending with infinitely many 9's. This restriction is needed, since (for example) $3_{b}=3$ for any $b>1$, whereas

$$
2.999 \cdots b=2+\frac{9}{b}+\frac{9}{b^{2}}+\frac{9}{b^{3}}+\cdots=2+\frac{9}{b-1} \neq 3
$$

unless $b=10$. Equation (3) is meaningful for some values of $a$ and $b \leq 1$, but to avoid exceptions we only consider $a, b>1$. In this range $a_{-} b$ is a binary operation for which 10 is both a left and right unit.

In fact, since the iterated subscripts can be grouped either from the bottom upwards or from the top downwards, there are really four sequences to be considered (it is convenient to index these sequences starting at 10): 


$$
\begin{aligned}
& (\alpha)=\left(\alpha_{10}, \alpha_{11}, \alpha_{12}, \ldots\right):=10,10 \_11,10 \_\left(11 \_12\right), 10 \_\left(11 \_\left(12 \_13\right)\right), \ldots, \\
& (\beta)=\left(\beta_{10}, \beta_{11}, \beta_{12}, \ldots\right):=10,10 \_11,\left(10 \_11\right) \_12,\left(\left(10 \_11\right) \_12\right) \_13, \ldots, \\
& (\gamma)=\left(\gamma_{10}, \gamma_{11}, \gamma_{12}, \ldots\right):=10,11 \_10,12 \_\left(11 \_10\right), 13 \_\left(12 \_\left(11 \_10\right)\right), \ldots, \\
& (\delta)=\left(\delta_{10}, \delta_{11}, \delta_{12}, \ldots\right):=10,11 \_10,\left(12 \_11\right) \_10,\left(\left(13 \_12\right) \_11\right) \_10, \ldots
\end{aligned}
$$

Sequence $(\alpha)$, for example, begins

$$
\begin{aligned}
& 10,10 \_11=11,10 \_\left(11 \_12\right)=10 \_13=13, \\
& 10 \_\left(11 \_\left(12 \_13\right)\right)=10 \_\left(11 \_15\right)=10 \_16=16, \\
& 10 \_\left(11 \_\left(12 \_\left(13 \_14\right)\right)\right)=10 \_\left(11 \_\left(12 \_17\right)\right)=10 \_\left(11 \_19\right)=10 \_20=20, \ldots
\end{aligned}
$$

The terms grow quite rapidly—see Table 1 These are now sequences A121263, A121265, A121295 and A121296 in [4].

Table 1 Initial terms of sequence $(\alpha),(\beta),(\gamma),(\delta)$.

\begin{tabular}{|r|r|r|r|r|}
\hline$n$ & $(\alpha)$ & $(\beta)$ & $(\gamma)$ & $(\delta)$ \\
\hline 10 & 10 & 10 & 10 & 10 \\
11 & 11 & 11 & 11 & 11 \\
12 & 13 & 13 & 13 & 13 \\
13 & 16 & 16 & 16 & 16 \\
14 & 20 & 20 & 20 & 20 \\
15 & 25 & 30 & 25 & 28 \\
16 & 31 & 48 & 31 & 45 \\
17 & 38 & 76 & 38 & 73 \\
18 & 46 & 132 & 46 & 133 \\
19 & 55 & 420 & 55 & 348 \\
20 & 65 & 1640 & 110 & 4943 \\
21 & 87 & 11991 & 221 & 22779 \\
22 & 135 & 249459 & 444 & 537226 \\
23 & 239 & 14103793 & 891 & 11662285 \\
24 & 463 & 5358891675 & 1786 & 46524257772 \\
25 & 943 & 19563802363305 & 3577 & 1092759075796059 \\
$\ldots$ & $\ldots$ & $\ldots$ & $\ldots$ \\
30 & 38959 & $3.6053 \ldots \times 10^{80}$ & 171999 & $2.5841 \ldots \times 10^{89}$ \\
$\ldots$ & $\ldots$ & $\ldots$ & $\ldots$ & $\ldots$ \\
35 & 9153583 & $8.6168 \ldots \times 10^{643}$ & 41795936 & $1.2327 \ldots \times 10^{8998}$ \\
$\ldots$ & $\ldots$ & $\ldots$ & $\ldots$ & $\ldots$ \\
100 & $4.0033 \ldots \times 10^{57}$ & $\ldots$ & $4.9144 \ldots \times 10^{114}$ & $\ldots$ \\
$\ldots$ & $\ldots$ & $\ldots$ & $\ldots$ & $\ldots$ \\
$\ldots$ & $6.8365 \ldots \times 10^{1098}$ & $\ldots$ & $3.4024 \ldots \times 10^{917}$ & $\ldots$ \\
& $a 109$ & & at $n=103$ & \\
\hline
\end{tabular}


In Theorem 1 we will show that, if $s_{n}$ is the $n^{\text {th }}$ term in any of the four sequences $(\alpha),(\beta),(\gamma)$ or $(\delta)$, indexed by $n=10,11, \ldots$, then

$$
\log \log s_{n} \sim n \log \log n \text { as } n \rightarrow \infty
$$

(in this paper all logarithms are to the base 10).

Since expressions like

$$
10^{11^{12^{13}}}
$$

are called towers, we will call expressions like those in (1) and $(\alpha),(\beta),(\gamma)$ or $(\delta)$, dungeons. For reasons that will be given in $\$ 2$, we believe that the standard parenthesizing of dungeons should be from the bottom upwards, and we will take this as the default meaning if the parentheses are omitted. For towers of exponents, parenthesizing from the top downwards is clearly better (for otherwise the tower collapses). The tower with $n^{\text {th }}$ term

$$
t_{n}:=10 \uparrow(11 \uparrow(12 \uparrow \cdots((n-1) \uparrow n) \cdots)), n=10,11, \ldots,
$$

(where $a \uparrow b$ denotes $a^{b}$ ) has the property that the iterated logarithm $\log ^{(n)} t_{n} \rightarrow \infty$ (note that $\log ^{(n)} t_{n}$ is well-defined for $n$ sufficiently large). When parenthesized from the bottom upwards, the tower with $n^{\text {th }}$ term

$$
u_{n}:=(\cdots((10 \uparrow 11) \uparrow 12) \cdots(n-1)) \uparrow n=10^{11 \cdot 12 \cdots \cdot n}, n=10,11, \ldots
$$

has the property that $\log \log u_{n} \sim n \log n$. Equation (5) shows that the dungeon sequences have a slower growth rate than either version of the tower.

In $\sqrt[3]{3}$ and $\$ 4$ we prove Theorem 1 and give some other properties of these sequences, such as the fact that sequence $(\alpha)$ converges 10-adically-for example, from a certain point on, the last ten digits are always ...9163204655.

In 45 we investigate the behavior as $n$ increases of the sequence with $n^{\text {th }}$ term $(n=1,2, \ldots)$

$$
a(n):=a_{-}\left(a_{-}\left(a_{-}\left(a_{-} \cdots a\right)\right)\right) \quad(\text { with } n \text { copies of } a)
$$


for a fixed real number $a>1$. If the parameter $a$ exceeds 10 this sequence certainly diverges, and for $a=10$ we have $a(n)=10$ for all $n \geq 1$. Somewhat surprisingly, it seems hard to say precisely what happens for $1<a<10$. The mapping from $a(n)$ to $a(n+1)=a_{a(n)}$ is a discrete dynamical system, which converges either to a single number (e.g. to the golden ratio if the parameter $a=1.1$ ), to a two-term limit cycle (e.g. if $a=1.05$ ) or diverges (e.g. if $a=\frac{100}{99}$ ). But we do not have a simple characterization of the parameters $a$ that fall into the different classes.

Section 2 contains some general properties of the subscript notation.

The following definition will be used throughout. If $a>1$ is a fixed real number with decimal expansion given by (2) and $x$ is any real number, we define the Laurent series

$$
L^{\langle a\rangle}(x):=\sum_{i=-\infty}^{k} c_{i} x^{i}
$$

so that $a_{-} b=L^{\langle a\rangle}(b)$. We use angle brackets to show the dependence on the parameter $a$. Note also that $L^{\langle a\rangle}(10)=a_{10}=a$ for all $a$.

Remark 1 . The choice of base 10 in this paper was a matter of personal preference.

Remark 2. To answer a question raised by some readers of an early draft of this paper, as far as we know there is no connection between this work and the basechanging sequences studied by Goodstein [2].

\section{Properties of the subscript notation}

In this and the following section we will be concerned with the numbers $a_{b}$ defined in (3) when $a$ and $b$ are integers $\geq 10$.

Lemma 1. Let $N=\sum_{i=0}^{k} v_{i} 10^{i}$, where the $v_{i}$ are nonnegative integers (not necessarily in the range 0 to 9 ), and suppose $b$ is an integer $\geq 10$. Then

$$
N_{b} \geq \sum_{i=0}^{k} v_{i} b^{i}
$$


Proof. If the $v_{i}$ are all in the range $\{0, \ldots, 9\}$ then the two sides of (8) are equal. Any $v_{i} \geq 10$, say $v_{i}=10 q+r, q \geq 1, r \in\{0, \ldots, 9\}$, causes the term $v_{i} b^{i}$ on the righthand side of $(8)$ to be replaced by $q b^{i+1}+r b^{i} \geq(10 q+r) b^{i}=v_{i} b^{i}$ on the left-hand side, and so the difference between the two sides can only increase.

Corollary 1. If $f(x)$ is a polynomial with nonnegative integer coefficients, and $b$ is an integer $\geq 10$, then $f(10)_{b} \geq f(b)$.

Lemma 2. Assume $a, b, a^{\prime}, b^{\prime}$ are integers $\geq 10$. Then

(i) $a^{\prime} \geq a$ if and only if $a_{b}^{\prime} \geq a_{b}$,

(ii) $b^{\prime} \geq b$ if and only if $a_{b^{\prime}} \geq a_{b}$,

(iii) $\left(a+a^{\prime}\right)_{b} \geq a_{b}+a_{b}^{\prime}$,

(iv) $a_{\left(b+b^{\prime}\right)} \geq a_{b}+a_{b^{\prime}}$,

(v) $a_{b} \geq \max \{a, b\}$.

Proof. (i) Suppose $a^{\prime}=\sum_{i=0}^{r^{\prime}} c_{i}^{\prime} 10^{i}>a=\sum_{i=0}^{r} c_{i} 10^{i}$, with all $c_{i}^{\prime}, c_{i} \in\{0, \ldots, 9\}$, and let $k$ be the largest $i$ such that $c_{i}^{\prime} \neq c_{i}$. Then $a_{b}^{\prime}-a_{b}=\sum_{i=0}^{k}\left(c_{i}^{\prime}-c_{i}\right) b^{i} \geq$ $b^{k}-\sum_{i=0}^{k-1} 9 b^{i}>0$. The converse has a similar proof. Claims (ii), (iv) and (v) are immediate, and (iii) follows from Lemma 1 .

Note that all parts of Lemma 2 may fail if we allow $a$ and $b$ to be less than 10 (e.g. $12_{2}=4<72=7 ; 6_{3}=6 \geq 6_{4}=6$, but $3<4$ ).

Lemma 3. Assume $a, b, c$ are integers $\geq 10$. Then

$$
\left(a_{-} b\right)_{-} c \geq a_{-}\left(b_{-} c\right) .
$$

Proof. The left-hand side of (9) is (in the notation of (7)) $L^{\langle a\rangle}\left(L^{\langle b\rangle}(10)\right)_{c}=\left(L^{\langle a\rangle} \circ\right.$ $\left.L^{\langle b\rangle}\right)(10)_{c}$, where $\circ$ denotes composition. The right-hand side is $L^{\langle a\rangle}\left(L^{\langle b\rangle}(c)\right)=$ $\left(L^{\langle a\rangle} \circ L^{\langle b\rangle}\right)(c)$, and the result now follows from Corollary 1 .

We can now explain why we prefer the "bottom-up" parenthesizing of dungeons. The reason can be stated in two essentially equivalent ways. First, $a_{-}\left(b_{-}\left(c_{-} d\right)\right)$, say, 
is simply

$$
L^{\langle a\rangle} \circ L^{\langle b\rangle} \circ L^{\langle c\rangle}(d)
$$

whereas no such simple expression holds for $\left(\left(a_{-} b\right)_{-} c\right) \_d$. To put this another way, consider evaluating the $n^{\text {th }}$ term of sequence $(\alpha)$ of $₫ 1$ To do this, we must repeatedly calculate values of $r_{S}$ where $r$ is $\leq n$ and $s$ is huge. But to find the $n^{\text {th }}$ term of $(\beta)$, we must repeatedly calculate values of $r_{s}$ where $r$ is huge and $s \leq n$. The latter is a more difficult task, since it requires finding the decimal expansion of $r$. Again, when computing the sequence $a(1), a(2), a(3), \ldots$ for a given values of $a$ (see (6)), as long as the terms are parenthesized from the bottom upwards, only one decimal expansion (of $a$ itself) is ever needed.

In 3 we will also need numerical estimates of $a_{b}$. If $a, b \geq 10$ then $a_{b}$ is roughly $10^{\log a \log b}$ (remember that all $\log$ arithms are to the base 10). More precisely, we have:

Lemma 4. Assume $a, b$ are integers $\geq 10$. Then

$$
10^{\lfloor\log a\rfloor\lfloor\log b\rfloor} \leq 10^{\lfloor\log a\rfloor \log b} \leq a_{b} \leq 10^{\log a \log b} .
$$

Proof. Suppose $a=\sum_{i=0}^{k} c_{i} 10^{i}$ where $k:=\lfloor\log a\rfloor, c_{i} \in\{0,1, \ldots, 9\}$ for $i=0,1, \ldots, k$, $c_{k} \neq 0$. The left-hand inequalities in 10 are immediate. For the right-hand inequality we must show that

$$
\sum_{i=0}^{k} c_{i} b^{i} \leq b^{\log a}
$$

or equivalently that

$$
\log \left\{c_{k} b^{k}\left(1+\sum_{i=0}^{k-1} \frac{c_{i}}{c_{k} b^{k-i}}\right)\right\} \leq(\log b)\left(\log \left\{c_{k} 10^{k}\left(1+\sum_{i=0}^{k-1} \frac{c_{i}}{c_{k} 10^{k-i}}\right)\right\}\right),
$$

and this is easily checked to be true using $b \geq 10$. 
3 Growth rate of the sequences $(\alpha),(\beta),(\gamma),(\delta)$

Theorem 1. If $s_{n}(n \geq 10)$ denotes the $n^{\text {th }}$ term in any of the sequences $(\alpha),(\beta)$, $(\gamma),(\delta)$ then

$$
\log \log s_{n} \sim n \log \log n \text { as } n \rightarrow \infty \text {. }
$$

Proof. From Lemma 4 it follows that

$$
\prod_{i=10}^{n}\lfloor\log i\rfloor \leq \log s_{n} \leq \prod_{i=10}^{n} \log i .
$$

For the upper bound, we have

$$
\log \log s_{n} \leq \sum_{i=10}^{n} \log \log i \leq n \log \log n .
$$

For the lower bound,

$$
\begin{gathered}
\log s_{n} \geq \prod_{i=10}^{n}\lfloor\log i\rfloor \geq \prod_{i=10}^{n} \log i\left(1-\frac{1}{\log i}\right), \\
\log \log s_{n} \geq \sum_{i=10}^{n} \log \log i-\sum_{i=10}^{n} \frac{1}{\log i},
\end{gathered}
$$

and the right-hand side is $\sim n \log \log n+O(n)$.

A slight tightening of this argument shows that there are positive constants $c_{1}, c_{2}$ such that

$$
n \log \log n-c_{1} \frac{n}{\log n}<\log \log s_{n}<n \log \log n-c_{2} \frac{n}{\log n}
$$

for all sufficiently large $n$.

Table 1 suggests that sequences $(\beta)$ and $(\delta)$ grow faster than $(\alpha)$ and $(\gamma)$. We can prove three of these four relationships.

Theorem 2. For $n \geq 10, \beta_{n} \geq \alpha_{n}$ and $\delta_{n} \geq \gamma_{n}$.

Proof. This follows by repeated application of Lemma 3

Lemma 5. If for some real number $k>10$ we have $a \geq k b$ and $\log c \geq \log k /(\log k-1)$, then $a_{-} c \geq k\left(c_{-} b\right)$. 
Proof. From Lemma 4 and the assumed bounds, we have

$$
\begin{aligned}
a_{-} c & \geq 10^{\lfloor\log a\rfloor \log c} \\
& \geq 10^{(\log a-1) \log c} \\
& \geq 10^{(\log b+\log k-1) \log c} \\
& =10^{(\log c)(\log k-1)} 10^{\log b \log c} \\
& \geq k\left(c_{-} b\right) .
\end{aligned}
$$

Theorem 3. For $n \geq 10, \beta_{n} \geq \gamma_{n}$.

Proof. From Table 1 this is true for $n \leq 23$. For $n>23$, since $\beta_{n+1}=\left(\beta_{n}\right)_{-}(n+1)$ and $\gamma_{n+1}=(n+1)_{-} \gamma_{n}$, the previous lemma (with $k=10^{4}$ ) gives us the result by induction.

\section{$4 p$-Adic convergence of the sequence $(\alpha)$}

For the next theorem we need a further lemma. Let us say that a polynomial $f(x) \in$ $\mathbb{Z}[x]$ is $m$-stable, for a positive integer $m$, if all its coefficients except the constant term are divisible by $m$. In particular, if $f(x)$ is $m$-stable, $f(x) \equiv f(0)(\bmod m)$.

Lemma 6. If the polynomial $f(x) \in \mathbb{Z}[x]$ is m-stable and the polynomial $g(x) \in \mathbb{Z}[x]$ is $n$-stable, then the polynomial $h(x):=f \circ g(x)$ is mn-stable.

Proof. If $f(x):=\sum_{i} f_{i} x^{i}, g(x):=\sum_{j} g_{j} x^{j}$, then $h(x)=\sum_{i} f_{i}\left(\sum_{j} g_{j} x^{j}\right)^{i}=\sum_{k} h_{k} x^{k}$ (say). When the expression for $h_{k}(k>0)$ is expanded as a sum of monomials, each term contains both a factor $f_{i}$ for some $i>0$ and a factor $g_{j}$ for some $j>0$.

Theorem 4. The sequence $\alpha_{10}, \alpha_{11}, \alpha_{12}, \ldots$ converges 10-adically.

Proof. We know from the above discussions that, for any $10 \leq k<n$, 


$$
\alpha_{n}=\Phi^{[k]}\left((k+1)_{-}(k+2)_{-}(k+3)_{-} \ldots \_n\right),
$$

where $\Phi^{[k]}(x)$ is the polynomial

$$
\Phi^{[k]}(x):=L^{\langle 10\rangle} \circ L^{\langle 11\rangle} \circ L^{\langle 12\rangle} \circ \cdots \circ L^{\langle k\rangle}(x)
$$

(We would normally write $\Phi_{k}(x)$, but since there are already two different kinds of subscripts in this paper, we will use the temporary notation $\Phi^{[k]}(x)$ in this proof instead.) Now $L^{\langle 20\rangle}(x), L^{\langle 21\rangle}(x), \ldots, L^{\langle 29\rangle}(x)$ are 2-stable and $L^{\langle 50\rangle}(x), \ldots, L^{\langle 59\rangle}(x)$ are 5-stable, so by Lemma $6 \Phi^{[59]}(x)$ is $10^{10}$-stable. This means that for $n \geq 60$, $\alpha_{n} \equiv \Phi^{[59]}(0)\left(\bmod 10^{10}\right)$, and so is a constant (in fact 5564023619) $\bmod 10^{10}$. Similarly, $L^{\langle 500\rangle}(x), L^{\langle 501\rangle}(x), \ldots, L^{\langle 509\rangle}(x)$ are 5-stable, so $\alpha_{n}$ is a constant mod $10^{20}$ for $n \geq 510$; and so on.

Remark 3. The same proof shows that $\alpha_{10}, \alpha_{11}, \alpha_{12}, \ldots$ converges $l$-adically, for any $l$ all of whose prime factors are less than 10.

\section{The limiting value of $a_{-} a_{-} a_{-} a_{-} \ldots$}

In this section we consider the behavior of the sequence $a(1), a(2), a(3), \ldots$ (see (6)) as $n$ increases, for a fixed real number $a$ in the range $1<a<10$. For example, we have the amusing identity

$$
{ }^{1.1} 1.1_{1.1_{1.1} 1_{1.1} 1_{1.1 .}}=\frac{1+\sqrt{5}}{2} .
$$

The sequence (6) is the trajectory of the discrete dynamical system $x \mapsto L^{\langle a\rangle}(x)$ when started at $x=a$. (Since $L^{\langle a\rangle}(10)=a$, we could also start all trajectories at 10.)

Suppose $a=\sum_{i=0}^{\infty} c_{i} 10^{-i}$ with all $c_{i} \in\{0,1, \ldots, 9\}$ and $c_{0} \neq 0$. The graph of $y=L^{\langle a\rangle}(x)$ is a convex curve, illustrated 1 for $a=1.1$ in Figure 1 which decreases monotonically from its value at $x=1$ (which may be infinite) and approaches $c_{0}$ as $x \rightarrow \infty$. This curve therefore meets the line $y=x$ at a unique point $x=\omega$ (say) in the

\footnotetext{
${ }^{1}$ This is a "cobweb" picture-compare Fig. 1.4 of [1].
} 
Fig. 1 Trajectory of $L^{<1.1>}(x)$ starting at $x=1.1$.

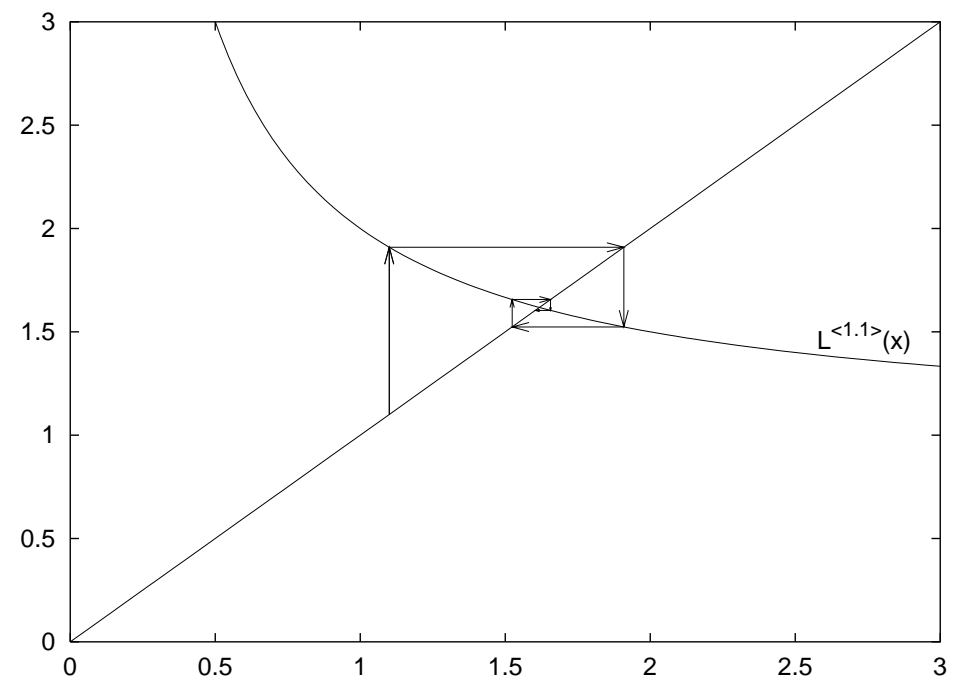

range $x>1$. The point $\omega$ is the unique fixed point for the dynamical system in the range of interest.

The general theory of dynamical systems [1], [3] tells us that the fixed point $\omega$ is respectively an attractor, a neutral point or a repelling point, according to whether the value of the derivative $L^{\langle a\rangle^{\prime}}(\omega)$ is between 0 and -1 , equal to -1 , or less than -1 . For our problem this does not tell the whole story, since we are constrained to start at $a$. However, since $L^{\langle a\rangle}(x)$ is a monotonically decreasing function, there are only a few possibilities. Cycles of length three or more cannot occur.

Theorem 5. For a fixed real number $a$ in the range $1<a<10$, and an initial real starting value $x>1$, consider the trajectory $x, L^{\langle a\rangle}(x), L^{\langle a\rangle} \circ L^{\langle a\rangle}(x), L^{\langle a\rangle} \circ L^{\langle a\rangle} \circ$ $L^{\langle a\rangle}(x), \ldots$. Then one of the following holds:

(i) $x=\omega$ is the fixed point, and the trajectory is simply $\omega, \omega, \omega, \ldots$,

(ii) the trajectory converges to $\omega$,

(iii) $x$ is in a two-term cycle, and the trajectory simply repeats that cycle,

(iv) the trajectory converges to a two-term limit cycle,

$(v)$ the trajectory diverges, alternately approaching 1 and $\infty$. 
Proof. If $a$ is an integer, then the trajectory is simply $x, a, a, a, \ldots$, and either case (i) or (ii) holds. Suppose then that $a$ is not an integer. Since $a$ is fixed, we abbreviate $L^{\langle a\rangle}$ by $L$ in this discussion, and write $L^{(k)}$ to indicate the $k$-fold composition of $L$, for $k=1,2, \ldots$. Because $L(x)$ is strictly decreasing, if $L^{(2)}(x)>x$, then $L^{(3)}(x)<L(x)$, $L^{(4)}(x)>L^{(2)}(x)>x$; if $L^{(2)}(x)<x$, then $L^{(3)}(x)>L(x), L^{(4)}(x)<L^{(2)}(x)<x$; and if $L^{(2)}(x)=x$, then $L^{(3)}(x)=L(x), L^{(4)}(x)=L^{(2)}(x)=x$. Hence if $x<L^{(2)}(x)$, then $x<L^{(2)}(x)<L^{(4)}(x)<\ldots$ and if $x>L^{(2)}(x)$, then $x>L^{(2)}(x)>L^{(4)}(x)>\ldots$ This means the even-indexed iterates form a monotonic sequence, so either converge or are unbounded, and similarly for the odd-indexed iterates. Eq. (4) implies that if the trajectory diverges then the lower limit must be 1 .

Note also that if $x<y<L^{(2)}(x)$, then $L^{(2 k)}(x)<L^{(2 k)}(y)<L^{(2 k+2)}(x)$, and if $x>y>L^{(2)}(x)$, then $L^{(2 k)}(x)>L^{(2 k)}(y)>L^{(2 k+2)}(x)$. So every $y$ between $x$ and $L^{(2 k)}(x)$ converges to the same limiting two-cycle as $x$ does, or diverges as $x$ does.

The following examples illustrate the five cases in the situation which most interests us, the trajectory $a, a_{-} a, a_{-}\left(a_{-} a\right), \ldots$ of $(6)$, that is, when we set $x=a$ in the theorem.

(i) This case holds if and only if $a$ is one of $\{2,3, \ldots, 9\}$.

(ii) Examples are $a=1+\frac{m}{10}$, for $m \in\{1, \ldots, 9\}$, when $\omega=(1+\sqrt{4 m+1}) / 2$ is an attractor (see (11)); $a=1+\frac{m}{100}$ for $m \in\{1,2,3\}$, when $\omega$, the real root $1.465 \ldots$, $1.695 \ldots$ or $1.863 \ldots$ of $x^{3}-x^{2}-m=0$ is an attractor; and $a=1+\frac{4}{100}$, when $\omega=2$ is neutral, but the trajectory still converges to $\omega$.

(iii) Examples are $a=1+\frac{m}{9}, m \in\{1, \ldots, 8\}, \omega$ is a neutral point, and the twoterm cycle is $\{a, 10\}$. (The trajectory does not include $\omega$.)

(iv) Examples are $a=1+\frac{m}{100}, m \in\{5, \ldots, 9\}, \omega$ is a repelling point, and the trajectory approaches a two-term limit cycle consisting of a pair of solutions to $L^{\langle a\rangle} \circ L^{\langle a\rangle}(x)=x$; also $a=1.1110000099, \omega$ is an attractor, but again the trajectory approaches a two-term cycle given by $L^{\langle a\rangle} \circ L^{\langle a\rangle}(x)=x$.

(v) Examples are $a=1+\frac{1}{10^{r}-1}, r \in\{2,3, \ldots\}, \omega$ is a repelling point, and the trajectory alternately approaches 1 or $\infty$. 
We do not know which values of $a$ fall into classes (ii) through (v). The distribution of the five classes for $1<a<10$ seems complicated.

\section{References}

1. R. L. Devaney, Dynamics of simple maps, in Chaos and Fractals, ed. R. L. Devaney and L. Keen, Proceedings Symposia Applied Math., Vol. 39, Amer. Math. Soc., Providence, RI, 1989.

2. R. L. Goodstein, On the restricted ordinal theorem, J. Symb. Logic, 9 (1944), 33-41.

3. H. A. Lauwerier, One-dimensional iterative maps, in Chaos, ed. A. V. Holden, Princeton Univ. Press, 1986, pp. 39-57.

4. N. J. A. Sloane, The On-Line Encyclopedia of Integer Sequences, published electronically at www.research.att.com/ njas/sequences/. 